\title{
Case Report on Familial Maxillomandibular Synostosis- A Rare Presentation
}

\section{Richa Chaudhary, Bhavana Lakhkar, Sachin Damke and Sham Lohiya}

Department of Paediatrics, Jawaharlal Nehru Medical College, Sawangi, Maharashtra, India

\section{Correspondence: \\ Dr Richa Chaudhary \\ Associate Professor \\ Department of Pediatrics \\ Jawaharlal Nehru Medical College, \\ Sawangi, \\ Maharashtra, India \\ Email: richa6101@gmail.com}

DOI: $10.3126 /$ jnps.v39i1.25992

Submitted on: $2019-10-20$

Accepted on: 2020-02-20

Acknowledgements: None

Funding: Nil

Conflict of Interest: None declared

Permission form IRB: Yes
To cite this article: Chaudhary $\mathrm{R}$, Iakhkar B, Damke S, lohiya S. Case report on Familial maxillomandibular synostosis- A rare presentation. J Nepal Paediatr Soc. 2019;39(1):49-52.

\section{ABSTRACT}

Congenital maxillomandibular synostosis is a rare congenital deformity associated with fusion between maxilla and mandible. We report a case of a five years old child who presented with inability to open her mouth since birth. Similar history was present in the elder sister. Diagnosis of familial congenital synostosis was made and was surgically managed.

Key words: case report; familial; maxillomandibular synostosis 


\section{INTRODUCTION}

Congenital syngnathia involving fusion of maxilla or zygoma with the mandible is an extremely rare condition. The fusion may vary from simple mucosal bands (synechiae) to complete bony fusion (synostosis). Mostly it is unilateral with incomplete fusion but may be bilateral. It may be in the midline or laterally. Congenital alveolar syngnathia may sometimes be associated with other congenital malformations like, cleft lip or cleft palate (Vander Woude Syndrome) where autosomal dominant inheritance is seen.1,2 Other syndromes associated with maxillomandibular syngnathia are popliteal pterygium syndrome, orofacial digital syndrome and cleft palate lateral synechiae syndrome. But isolated congenital syngnathia, without other malformation is another phenotype with unknown molecular basis. It is a very rare condition with only few cases reported till now of familial maxillomandibular synostosis and presentation of such case reports will guide the health care providers dealing with children to manage these cases better as early surgical intervention followed by oral dilatation exercise is associated with good functional outcome. We report a rare case of familial maxillomandibular synostosis, where two siblings presented with bilateral synostosis.

\section{CASE REPORT}

A five years old female child presented in the OPD of the paediatric department with the history of not being able to open her mouth properly since birth. There was also history of feeding difficulty and not being able to vocalise properly. In the treatment history, there was history of some procedure done at the age of one year, after which there was some mouth opening but later there was refusion. There was similar history in the elder sibling who was six

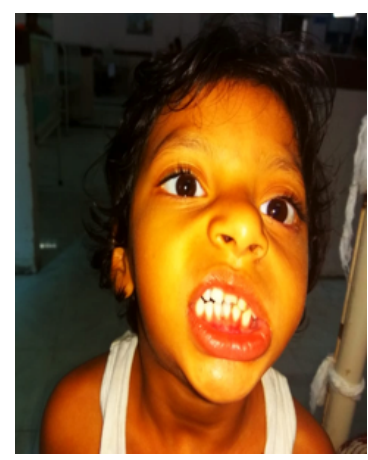

Figure 1. Before surgery years old female child along with history of third degree consanguinity in the parents.

On examination, there was complete fusion of upper and lower jaw on both the sides of the mouth posteriorly. There was some space between the two bones anteriorly through which parents used to feed the child with lot of difficulty. The rest of the physical examination was within normal limits with no associated malformations. There were similar findings in the elder sister. 2D ECHO and USG abdomen was done to rule out associated malformations which were normal. Patient was posted for surgical release of bilateral maxillomandibular complex. Fiberoptic nasotracheal intubation was done and general anesthesia (GA) was induced. Bony mass was released bilaterally and both the jaws were separated. Bilateral coronoidotomy was carried out, active mouth opening was achieved up to $25 \mathrm{~mm}$. Post operatively recovery was uneventful. Patient was shifted to oral liquid diet and was discharged after seven days on regular liquid diet. Patient on follow up did not have satisfactory mouth opening with fibrous band formation on right side and was again taken for Brismans Mouth opening procedure. Under GA unilateral fibrous bands were released from molar region to retromolar region on right side. Release of bony fusion was also done on right side, and active mouth opening of $30 \mathrm{~mm}$ was achieved. Post operatively recovery was uneventful.

Patient was advised oral dilatation exercise daily and mouth opening of $45 \mathrm{~mm}$ was achieved on discharge with advice to continue the exercise. On follow-up the opening was maintained and child

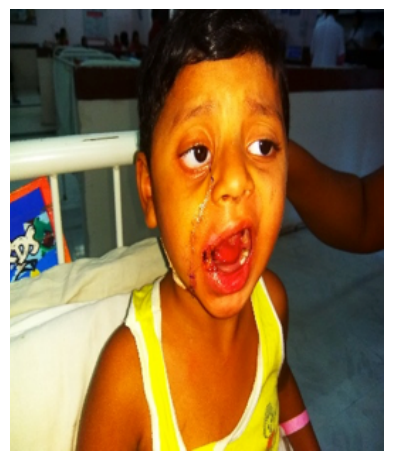

Figure 2. Post- operatively the child was able to open her mouth fully 
was doing well on liquid diet. The same procedure has been planned for the other sibling.

\section{DISCUSSION}

Limited mouth opening due to fusion of the maxilla and mandible (Syngnathia) are a rare group of anomalies. It may be bony fusion or may have only fibrous attachment. Occurrence may be unilateral or bilateral, partial or complete. ${ }^{1,2}$ Isolated occurrence of bony syngnathia (synostosis) is a very rare condition with few reported cases.

Goodacre and Wallace ${ }^{3}$ have proposed some of the etiological hypothesis for congenital bony fusion which include persistence of buccopharyngeal membrane, amniotic constriction bands in the region of the developing first branchial arch, environmental insults, drugs such as meclozine and large doses of Vitamin A. Dawson et al. reported that congenital syngnathia are not associated with history of consanguinity in the parents, any drug or toxin exposure and there is no familial tendency. ${ }^{4}$ Mir et al. and Poovazhagi et al. reported cases which revealed a history of consanguinity and in our case there is both history of consanguinity and familial tendency. 5,6

Our patient presented in paediatric outpatient department with maxillomandibular fusion present since birth. The baby was not able to open her mouth, vocalise and feed normally. Congruent with the observation that congenital bony fusion is clinically diagnosed soon or after birth as the neonate presents with airway and feeding difficulties depending upon the severity of fusion. (However, our case did not have any problems at birth) Child with complete bony fusion bilaterally will present immediately after birth and physician taking care should be aware of the condition and airway should be managed immediately. Feeding is the other issue in these patients which is addressed with RT feeding in infants with complete fusion. Early surgical release should be undertaken as it interferes with the development of the Jaw and ankylosis of the temporomandibular joint (TMJ) may occur due to restriction of functional movements in the growing stage. Various studies have shown that functional results following surgery are very good in isolated syngnathia and hence early surgical removal of maxillomandibular fusion should be the standard protocol. Following surgery active physical therapy is of utmost importance as there are chances of refusion as happened in our case. Acceptable mouth opening is achieved one to two weeks after surgery. Parents should be taught the manual exercise techniques before discharge.

\section{CONCLUSIONS}

Congenital synostosis is a very rare condition and pose difficulty in feeding, reduced mouth opening, and also affects the development of the jaws, if not excised at an early age. Early diagnosis and timely management of such cases is associated with very good outcome and hence we hope this case report will help the primary care physician to manage this conditional appropriately with good functional outcome.

\section{REFERENCES}

1. Kondo S, Schutte BC, Richardson RJ, Bjork BC, Knight AS, Watanabe Y et al. Mutations in IRF6 cause Van der Woude and popliteal pterygium syndromes. Nat Genet. 2002;32(2):285-9. DOI: 10.1038/ng985.

2. Ferrero GB, Baldassarre G, Panza E, Valenzise M, Pippucci T, Mussa A et al. A heritable cause of cleft lip and palate - Van der Woude syndrome caused by a novel IRF6 mutation. Review of the literature and of the differential diagnosis. Eur J Pediatr. 2010;169(2):223-8. DOI:10.1007/s00431-009-1011-3

3. Goodacre TE, Wallace AF. Congenital alveolar fusion. Br J Plast Surg. 1990 Mar; 43(2): 203-209. DOI: $10.1016 / 0007-1226(90) 90162-\mathrm{s}$ 
4. Dawson KH, Gruss JS, Myall RW. Congenital bony syngnathia: a proposed classification. Cleft Palate Craniofac J. 1997 Mar; 34(2):141-6. DOI:10.1597/1545-1569_1997_034_0141_cbsapc_2.3.co_2

5. Poovazhagi V, Vijayakumar V, Kumudha J, Balachandran K. Congenital fusion of maxilla and mandible (bony syngnathia). Pediatric Oncall. 2009 Dec;6(12):63.

6. Mir M, Iqbal S, Hafeez A, Zargar H, Rasool A, Mohsin M, et al. Syngnathia without any other associated anomaly: A very rare case report. Int J of Plastic Surg. 2006;4(1). 